Open Access

\title{
Erratum to: Overview of business innovations and research opportunities in blockchain and introduction to the special issue
}

J. Leon Zhao ${ }^{1 *}$, Shaokun Fan² and Jiaqi Yan ${ }^{3}$

* Correspondence:

jlzhao@cityu.edu.hk

${ }^{1}$ City University of Hong Kong,

Hong Kong, China

\section{Erratum}

After publication of the original article (Zhao et al., 2016), the author has noticed an error in the following sentence of the second paragraph in Concluding Remarks section:

"For instance, while the original value of blockchain is to decentralize organizations by running Bitcoin transactions with a bank, new blockchain applications are more likely applied in centralized or partially centralized organizations such as a national bank."

The word 'with' should be corrected to 'without'.

We apologize for any inconvenience caused.

Author details

${ }^{1}$ City University of Hong Kong, Hong Kong, China. ${ }^{2}$ Oregon State University, Corvallis, USA. ${ }^{3}$ Nanjing University, Nanjing, China.

Received: 8 May 2017 Accepted: 8 May 2017

Published online: 16 May 2017

\section{Reference}

Zhao JL et al (2016) Overview of business innovations and research opportunities in blockchain and introduction to the special issue. Financial Innovation 2:28. doi:10.1186/s40854-016-0049-2 